\title{
Phase diagram of octapod-shaped nanocrystals in a quasi-two-dimensional planar geometry
}

\author{
Weikai Qi, ${ }^{1}$ a) Joost de Graaf, ${ }^{1}$ Fen Qiao, ${ }^{2}$ Sergio Marras, ${ }^{2}$ Liberato Manna, ${ }^{2}$ \\ and Marjolein Dijkstra ${ }^{1, b)}$ \\ ${ }^{1}$ Soft Condensed Matter, Debye Institute for Nanomaterials Science, Utrecht University, Princetonplein 5 , \\ 3584 CC Utrecht, The Netherlands \\ ${ }^{2}$ Istituto Italiano di Tecnologia (IIT), Via Morego 30, 16163 Genova, Italy
}

(Received 7 December 2012; accepted 18 March 2013; published online 15 April 2013)

\begin{abstract}
Recently, we reported the formation of crystalline monolayers consisting of octapod-shaped nanocrystals (so-called octapods) that had arranged in a square-lattice geometry through drop deposition and fast evaporation on a substrate [W. Qi, J. de Graaf, F. Qiao, S. Marras, L. Manna, and M. Dijkstra, Nano Lett. 12, 5299 (2012)]. In this paper we give a more in-depth exposition on the Monte Carlo simulations in a quasi-two-dimensional (quasi-2D) geometry, by which we modelled the experimentally observed crystal structure formation. Using a simulation model for the octapods consisting of four hard interpenetrating spherocylinders, we considered the effect of the pod length-to-diameter ratio on the phase behavior and we constructed the full phase diagram. The methods we applied to establish the nature of the phase transitions between the various phases are discussed in detail. We also considered the possible existence of a Kosterlitz-Thouless-type phase transition between the isotropic liquid and hexagonal rotator phase for certain pod lengthto-diameter ratios. Our methods may prove instrumental in guiding future simulation studies of similar anisotropic nanoparticles in confined geometries and monolayers. () 2013 American Institute of Physics. [http://dx.doi.org/10.1063/1.4799269]
\end{abstract}

\section{INTRODUCTION}

Recent advances in the synthesis of colloids and nanoparticles have made possible the creation of monodisperse samples consisting of complex particles with anisotropic hard and soft interactions. ${ }^{1-8}$ Such samples were used to experimentally study self-assembly and mesophase behavior. ${ }^{6-11}$ Concurrently, new simulation techniques were developed to explain the experimentally observed phenomenology and to tackle the complex numerical problems that such investigations bring about. ${ }^{8,12-23}$ Most of these simulation studies focussed on convex particles in two- and three-dimensional (2D and 3D) systems, see Refs. 7, 9, 10, 12-14, 18, and 20-22 among others. However, in only a limited number of studies the behavior of nonconvex anisotropic particles was considered. $6,8,11,15-17,19,23,24$

The phase behavior of particles in 2D or quasi-2D systems, i.e., 3D particles in a 2D monolayer, is often dissimilar from that of particles in 3D systems. ${ }^{25,26}$ The study of particle monolayers therefore offers many opportunities for the creation of new materials with bulk properties that differ substantially from the materials that form by $3 \mathrm{D}$ self-assembly. ${ }^{27,28}$ This has led to a strong experimental and simulation interest in the behavior of (anisotropic) particles in (quasi-)2D. The mesophase behavior of convex anisotropic particles in quasi2D, such as rods and polygonal (e.g., square and pentagonal) particles, has for instance been studied in experiments and by simulations. ${ }^{29-38}$

\footnotetext{
a)Electronic mail: W.Qi@uu.nl

b)Electronic mail: M.Dijkstra1@uu.nl
}

For rods, theoretical investigations ${ }^{29,31}$ suggested a possible Kosterlitz-Thouless (KT) isotropic-nematic phase transition, when the aspect ratio exceeded 7.0, and its existence was confirmed by simulations and experiments. ${ }^{30-32}$ For hard squares and rectangles, a tetratic phase with quasi-long-range orientational order was observed in simulations. ${ }^{34,36}$ Contrary to the simulation results, a hexagonal plastic-crystal (rotator) phase appeared in experiments of square colloidal particles under confinement. ${ }^{37}$ This finding could be explained by the roundness of particles. ${ }^{38}$

Despite this strong interest in (quasi-)2D systems, there are still many unanswered questions, even for a relatively simple system consisting of monodisperse hard disks. For this system a two-step continuous solid-liquid phase transition, via a hexatic phase, is predicted by theory ${ }^{39-41}$ However, other melting mechanisms cannot be excluded. ${ }^{42}$ The recent interest in nonconvex particles, such as L- and cross-shaped particles, ${ }^{17}$ crescent-shaped particles,${ }^{23}$ as well as octapods, ${ }^{8}$ in quasi-2D geometries, presents further challenges for simulation studies of these systems. The main problem stemming from the geometric restrictions and the complex interactions that arise between such particles has yet to be resolved. ${ }^{43}$

Our group recently reported an experimental and simulation study of the formation - by solvent deposition and evaporation - of monolayers on a substrate consisting of octapod-shaped nanocrystals that arranged into a squarelattice crystal. ${ }^{8}$ This study was a direct continuation of the self-assembly experiments in 3D, that showed hierarchical interlocking-chain and superstructure formation, which was induced by the octapod's shape and shape-induced van der 
Waals (vdW) interactions. ${ }^{6}$ In this paper we give an extensive overview of the simulation techniques used to analyse the observed crystal structure formation in the quasi-2D monolayers of octapods.

We considered a simple model for the octapods, consisting of four hard, interpenetrating spherocylinders. These particles were constrained to move in a planar quasi-2D geometry, namely that of the monolayers on a substrate observed in the experimental system. ${ }^{8}$ Using floppy-box Monte Carlo (FBMC) simulations, ${ }^{16,44,45}$ we constructed the highpressure crystal structures for these octapods as a function of the pod length-to-diameter ratio $L / D$, with $L$ the length and $D$ the diameter. Subsequent isothermal-isobaric (NPT) and isothermal-isochoric ( $N V T)$ Monte Carlo (MC) simulations were used to establish the equations of state (EOSs) for several conveniently chosen $L / D \in[0.0,8.0]$. By employing order parameters and free-energy calculations the full phase diagram for the hard-octapod model could be established.

Our results show that the high-density phase is a rhombic crystal (RC) for $L / D \in[0.0,1.7]$, a square-lattice crystal (SC) for $L / D \in[1.8,5.0] \cup[6.3,8.0]$, and a binary-lattice square crystal (BSC) for $L / D \in[5.1,6.2]$. These results appear to be consistent with the experimental observations. ${ }^{8}$ For $L / D \in[0.0,1.7]$ we found that the RC melted into an isotropic-liquid (IL) phase, via a hexagonal plastic-crystal (rotator) phase (HR). In the region $L / D \in[1.7,2.0]$ there appears to be a three-step phase transition: by reducing the pressure the SC melted into the RC, which subsequently melted into the HR phase, and finally the HR phase melted into the IL phase. For specific values of $L / D$ in this region, the algebraic decay of the bond-orientational correlation function hints at a possible Kosterliz-Thouless-type (KT-type) ILHR phase transition. We confirmed that for $L / D>2.0$ the SC melted into an IL phase via a first-order phase transition using free-energy calculations and direct (coexistence) isothermal-isochoric $(N V T)$ simulations. In the range $L / D$ $\in[5.1,6.2]$ the BSC was found to be stable at high pressures and found to melt into the SC for lower pressures, which subsequently melted into the IL phase via a first-order phase transition upon lowering the pressure further. A continuous phase transition between the BSC and SC was observed, for which only the relative orientations of the particles in the BSC changed, but the crystal lattice itself remained unaffected.

This paper is structured as follows. Section II briefly introduces the experimental observations. In Sec. III, we present the model and the simulation techniques we use to study the experimental findings. In Secs. IV and V we predict the candidate crystal structures for the octapods at high packing fractions and present the phase diagram that we obtained using these structures, respectively. Section VI summarizes the research presented in this paper and gives an outlook. Finally, we give further details on the freeenergy calculations and the order parameters that we used in Appendices A and B, respectively. In Appendix C, we present a free-volume theory for our system, by which we verified our Monte Carlo simulation results at high densities in the SC phase.

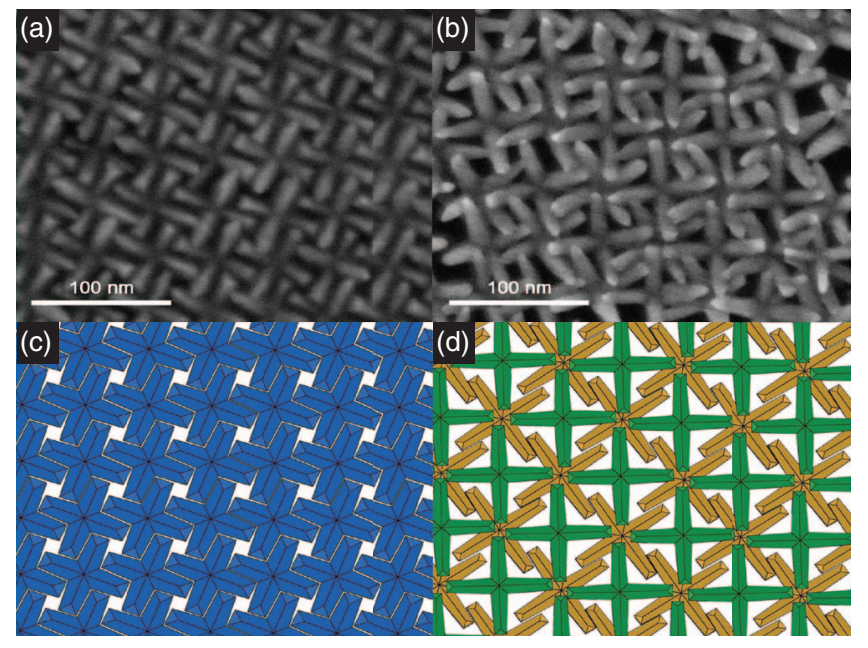

FIG. 1. High-resolution SEM secondary electron images (SEI) and related models showing the influence of the length-to-diameter ratio $(L / D)$ on the organization of the octapods. ${ }^{8}$ (a) and (c) For $L / D=4.8$ only simple squarelattice crystals were formed, while (b) and (d) for $L / D=5.9$ binary-lattice square crystals were occasionally found, as indicated by the outline in (b). The scale bars are $100 \mathrm{~nm}$.

\section{EXPERIMENTS}

The octapod-shaped nanocrystals used in the experiments were synthesized according to literature procedures. ${ }^{5,6,8} \mathrm{~A}$ freshly prepared solution of octapods in toluene was dropcast on various substrates, after which the solvent was allowed to evaporate at room temperature, see Ref. 8 for the full details. In the samples two types of crystalline monolayers were found in the inner regions of the area delimited by the coffee stain that resulted from the solvent evaporating. For octapods with an average pod length-to-diameter ratio of $L / D$ $\approx 4.8$, square-lattice crystals ( $\mathrm{SCs}$ ) were observed, see Fig. 1(a). When the pod length was larger, on average $L / D$ $\approx 5.9$, we found evidence of binary-lattice square crystals (BSCs), see Fig. 1(b), in addition to SCs.

\section{SIMULATIONS AND MODEL}

To study the experimental findings using Monte Carlo (MC) simulations, we modelled the octapod-shaped nanocrystals by four hard interpenetrating spherocylinders. These spherocylinders intersect in their respective centres and are oriented along the $( \pm 1, \pm 1, \pm 1)$ directions of a standard Cartesian coordinate frame (the origin is located in the intersection point). The octapod model is completely described by setting the length-to-diameter ratio $L / D$, with $L$ the length (excluding the hemispherical caps) and $D$ the diameter. In the simulations we set $D=1.0$ and let $L$ vary between 0.0 and 8.0. Figure 2(a) shows our definition of $L$ and $D$ and Fig. 2(b) shows our model for several choices of $L / D$. Note that for $L / D$ $=0.0$ the model reduces to a sphere.

In order to emulate the quasi-2D geometry of the monolayers observed in the experimental system, we constrained the centres of our octapod models to move in the $x y$-plane and imposed that the tips of the pods are coplanar with their centres. Effectively the octapods are sandwiched between two frictionless walls, with four tips touching the top and four tips 
(a)

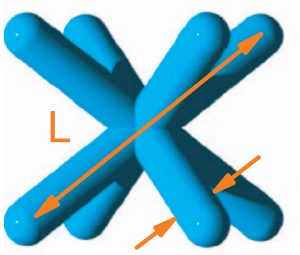

D

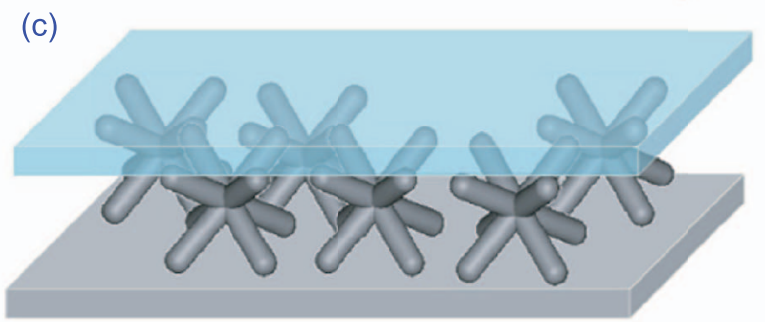

FIG. 2. (a) An example of the hard octapod model, which consists of four interpenetrating spherocylinders with a length-to-diameter ratio $L / D=6.0$. The orange arrows indicate the length $L$ and diameter $D$ definition we used for our model. (b) The model for several of the values of $L / D$ that we considered in this paper. (c) An illustration of the octapod model in the quasi-2D geometry that we used. We constrained the bottom four tips to be in contact with the substrate; the octapods therefore effectively behave as if they are trapped between two frictionless walls.

touching the bottom wall (substrate), see Fig. 2(c). The simulation box has $2 \mathrm{D}$ periodicity in the direction of the plane and is not periodic in the direction perpendicular to it. In the isothermal-isochoric $(N V T)$ ensemble we only allowed translations in the $x y$-plane and rotations around the $z$-axis. For simulations in the isothermal-isobaric (NPT) ensemble we used the same particle moves and allowed the box to change its size and shape in the $x y$-plane.

We define the reduced pressure as $P^{*}=P D^{2} / k_{B} T$ where $k_{B}$ is Boltzmann's constant, $T$ is the temperature, and $P$ is the (2D) pressure. We express the density in terms of this area as well $\rho=N / A$, with $N$ the number of particles. However, the volume fraction occupied by the particles is defined as $\eta=\rho V_{p} / h$, where $h$ is the distance between the two confining walls and $V_{p}$ is the volume of the octapod. We determined the height $h=L / \sqrt{3}+D$ using simple geometric arguments and $V_{p}$ using Monte Carlo integration, for which we achieved a numerical precision of 4 decimals.

Finally, we mention that we chose not to model the octapods using the triangular-tessellation mesh of Ref. 16. The reason for this is that the simpler spherocylinder-based model enables us to achieve greater computational efficiency and thereby allows us to study a greater number of particles in a reasonable amount of time, while still giving a good approximation for the shape of the particle.

\section{DENSE-PACKING CRYSTAL STRUCTURES}

We performed floppy-box Monte Carlo (FBMC) simulations in the spirit of Refs. 16, 44, and 45 to determine candidate crystal structures for the octapod models in the quasi-2D geometry. Our experimental results led us to conclude that the observed crystal structures were probably induced by the geo-
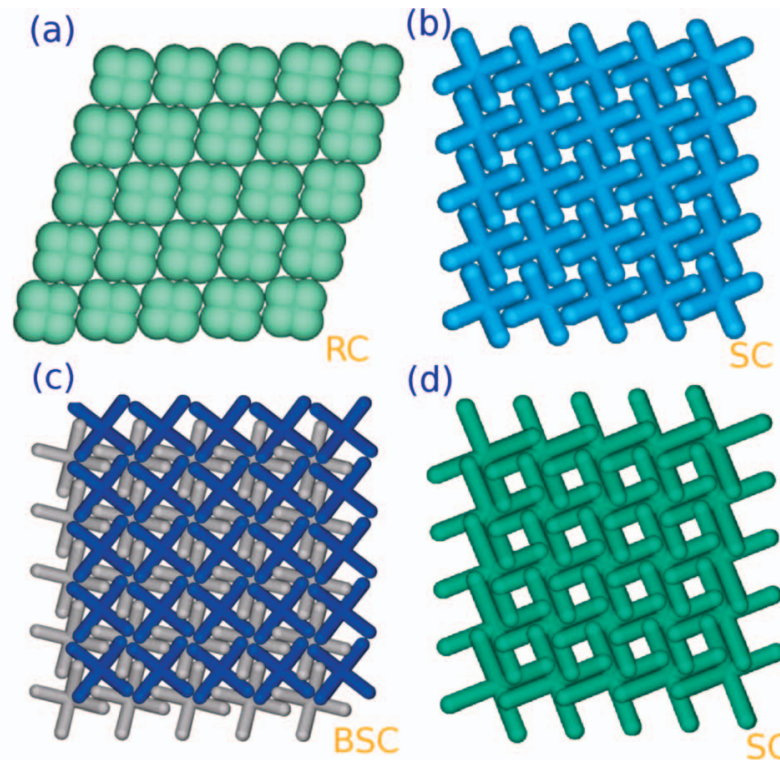

(d)
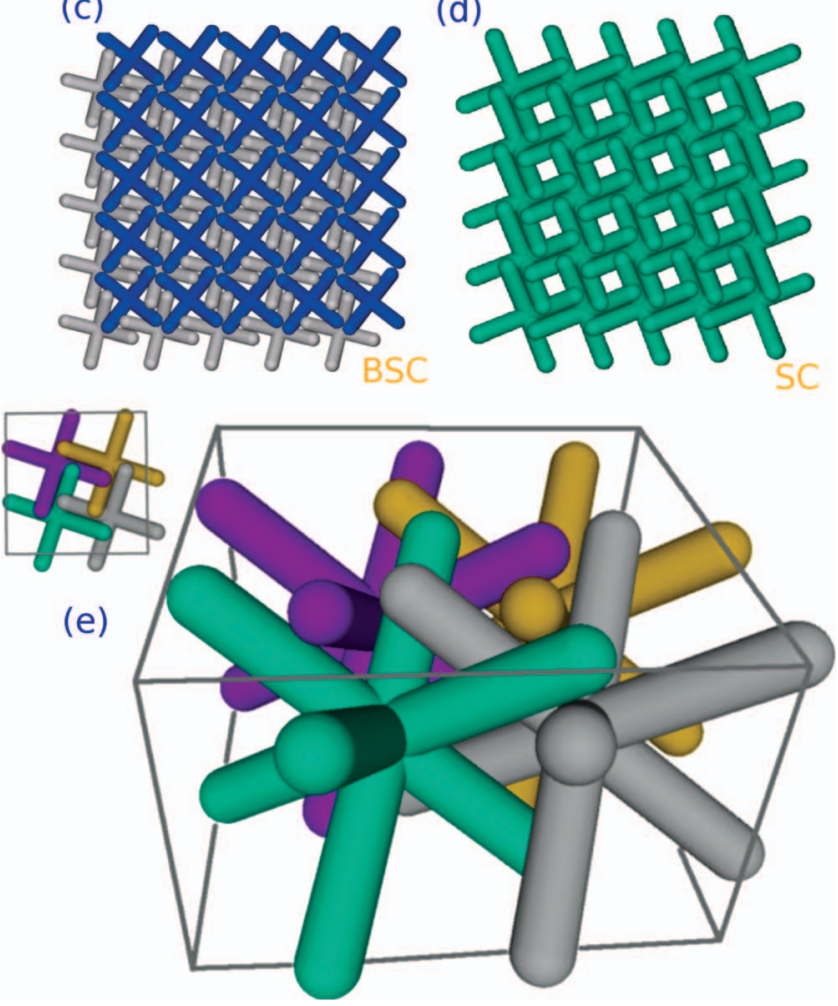

FIG. 3. Top views of quasi-2D densely packed structures obtained for different length-to-diameter ratios (L/D) of the hard octapod model. (a) A rhombic crystal (RC) for $L / D=1.0$. (b) A square crystal (SC) for $L / D=4.0$, which is not interlocking. (c) A binary-lattice square crystal (BSC) for $L / D=6.0$. The different orientations of the particles in the two sublattices are illustrated by the use of color. Note that the total lattice is again square, hence the name binary-lattice square crystal. (d) Another SC, for which the octapods are interlocking $(L / D=7.0)$. (e) A $3 \mathrm{D}$ image showing four octapod models in an interlocking configuration, the octapods are indicated with different colors for clarity. The inset shows a top view of the 3D image, in which the arms of the interlocking octapods appear to overlap.

metric constraints that the hard core of the octapods imposed on the structures these nanocrystals can form. ${ }^{8}$ We therefore assumed that the van der Waals ( $\mathrm{vdW}$ ) interactions between octapods ${ }^{6}$ are dominated by the aggregation forces that occur during solvent evaporation, thereby allowing for an accurate description using a hard-particle model.

We used $N=1, \ldots, 6$ particles in a unit cell and slow pressure annealing from a value of $P^{*} \approx 0.5$, for which the system behaved liquid-like $(\eta=0.01)$, to $P^{*} \approx 2,500$ to achieve crystallization. We performed compression runs a total of 100 times, for roughly 100 values of $L / D \in[0.0,8.0]$. For each value of $L / D$ we selected the densest packings and we determined their crystal structure. See Fig. 3 for a visual representation of the three different high-density crystal 

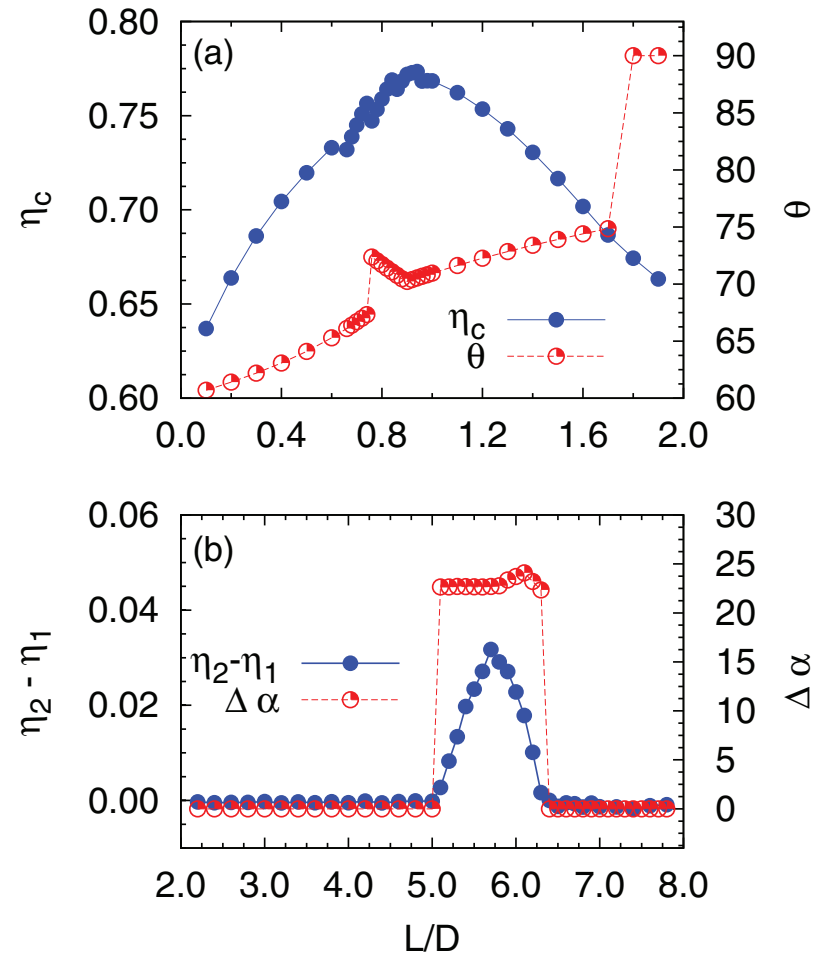

FIG. 4. (a) Solid blue circles show the "maximum" packing fraction $\eta_{c}$ as a function of $L / D<2.0$. Open red circles show the angle $\theta$ (in degrees) between the two lattice vectors in $x y$-plane that span the unit cell of the crystal structure. (b) Solid blue circles show the difference in the packing fraction $\eta_{2}-\eta_{1}$ between the square crystal (SC, one particle in the unit cell, $\eta_{1}$ ) and the binary-lattice square crystal (BSC, two particles in the unit cell, $\eta_{2}$ ) as a function of shape factor $L / D$. We only observed the BSC phase for $L / D$ $\in[5.0,6.3]$, i.e., $\eta_{2}-\eta_{1} \neq 0$ in this region. Open red circles show the angular difference $\Delta \alpha$ (in degrees) between the orientation of the particles in the two sublattices of the BSC.

structures that we obtained: rhombic crystals (RCs), squarelattice crystals (SCs), and binary-lattice square crystals (BSCs). The BSC has the unusual property that the lattice structure is the same as that of the SC, but that it is decomposed into two sublattices, for which the particles have different orientations, see Fig. 3(d). Note that in a top view of the simulation system (the viewpoint we typically use) the nonconvex nature of the $3 \mathrm{D}$ octapod model in the quasi-2D system can lead to an "optical illusion" where it seems that the particles overlap, when they in fact do not. This phenomenon can be explained by a type of interlocking of the octapods, as is illustrated in Fig. 3(e). This interlocking is different from the interlocking observed for octapods in the chains that formed in solution. ${ }^{6}$ Throughout this paper we refer to the arrangement in Fig. 3(e) as "interlocking."

We also determined the "maximum" packing fraction $\eta_{c}$, see Fig. 4(a). The discontinuities in the region $L / D \in[0.6$, 1.0] can be attributed to the nonconvex nature of the octapods; however, there is only one type of crystal structure in this region. To aid in establishing the nature of the crystal structures in the region $L / D \in[0.0,2.0]$ we considered the angle $\theta$ between the lattice vectors in the $x y$-plane that span the unit cell, see Fig. 4(a). We found a rhombic crystal (RC) phase for $L / D<1.7$. The RC has a deformed triangular morphology (its space group is pmm), and the angle between two lattice vec- tors ranges from $60^{\circ}$ to $67.5^{\circ}$. Our results for $L / D<1.7$ are reminiscent of the results obtained in experiments and simulations of rounded square particles. ${ }^{37,38}$ This correspondence can be explained by the shape of the octapods for these values of $L / D$, since the particles have an interaction cross section that is roughly a rounded square with concave edges. For octapod-shaped nanoparticles there are also experimental indications that a rhombic monolayer can form when the pod length-to-diameter ratio is small. ${ }^{46}$

To determine the range in which the (B)SC structures are found (at high pressure) we calculated the angular difference $\Delta \alpha$ between the orientation of neighboring octapods, see Fig. 4(b). The orientational difference between neighboring octapods for the particles in the BSC is $\Delta \alpha=22.5^{\circ} \pm 1^{\circ}$, also see Fig. 4(b), whereas for the SCs $\Delta \alpha=0$. Octapods with $L / D$ $\in[5.1,6.2]$ can form both the BSC and the SC phase, but the BSC phase achieves a higher packing fraction and is therefore favoured at higher pressures. By combining the aforementioned results we obtained the following subdivision for the high-density structures: RCs for $L / D \in[0.0,1.7]$, SCs for $L / D \in[1.8,5.0] \cup[6.3,8.0]$, and BSCs for $L / D \in[5.1,6.2]$.

The SCs that were found for octapods with $L / D \in[1.8$, 5.0] differ from those in the range $L / D \in[6.3,8.0]$. For $L / D$ $<5.0$ the pods of the octapods in the SC are alongside each other, i.e., there is no interlocking, whereas for $L / D>6.3$ the octapods are interlocking. This is illustrated by the pods appearing to overlap in Fig. 3(d). This interlocking of the octapods effectively improves the packing fraction and at the same time makes it harder for the structure to be deformed. In the BSC particles are also interlocked with each other. Finally, it should be mentioned that our SC result for $L / D$ $\approx 4.8$ indeed corresponds to the experimental observation, see Fig. 1(a), and that BSC fragments were also recovered in the experiments for $L / D \approx 5.9$, see Fig. $1(\mathrm{~b})$.

\section{PHASE DIAGRAM AND EQUATIONS OF STATE}

Using our high-density crystal structures, we were able to study the phase behavior of our hard octapod models in the quasi-2D geometry. We used NPT variable-box-shape simulations to obtain the crystal branch of the equation of state (EOS), as well as any mesophases, by melting the highdensity crystal. We employed regular NPT simulations to establish the isotropic-liquid (IL) branch of the EOS by compressing from a dilute system of octapods. To obtain accurate results we used 400-900 particles in the simulation box. We determined the EOS for several conveniently chosen $L / D$ $\in[0.0,8.0]$ and used these to construct the phase diagram.

Figure 5(a) shows the EOS for octapods with $L / D=4.0$ in the quasi-2D system, for which there is a first-order ILSC phase transition. It proved problematic to accurately establish the phase boundaries using the EOS only. We did not observe crystallization from the IL phase because compression of the octapods from a dilute phase always resulted in a system that became disordered and jammed (glass-like) at high pressures. We did observe melting from the SC phase, but we suspect that the nonconvex nature of the octapods allows the crystal to be significantly superheated before melting occurs. Therefore, we used free-energy calculations to 


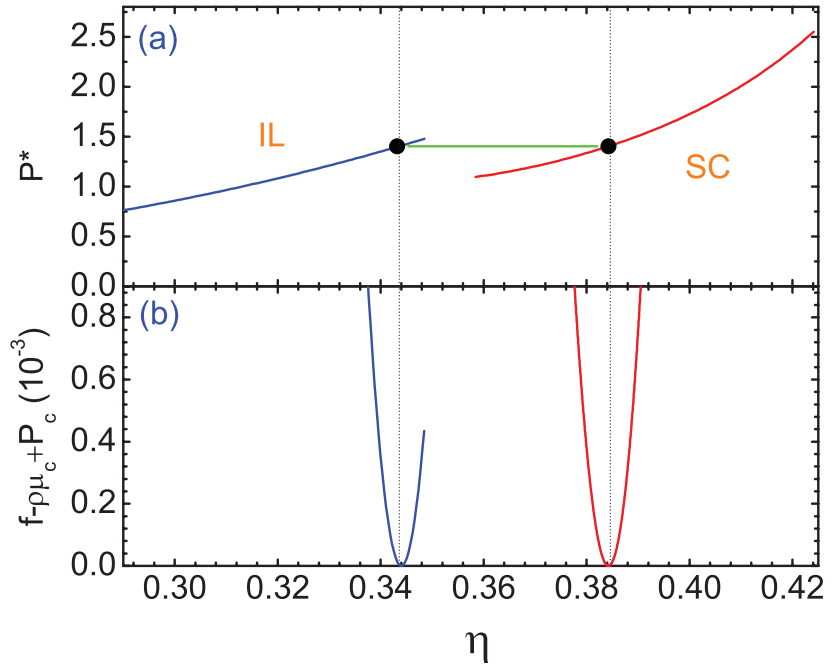

FIG. 5. (a) The equation of state (EOS) near the coexistence region for octapods with $L / D=4.0$ in the planar quasi-2D geometry. Here $P^{*}=P D^{2} / k_{B} T$ is the reduced pressure, with $P$ the (2D) pressure, $k_{B}$ Boltzmann's constant, $T$ the temperature, and $D$ the diameter of the pods. We also define $\eta=\rho V_{p} / h$, where $h=L / \sqrt{3}+D$ is the height of the octapods and $V_{p}$ is their volume. The green line and black points show the coexistence pressure and densities of the square-lattice crystal (SC) and isotropic-liquid (IL) phase. (b) The reduced free energy $f-\rho \mu_{c}+P_{c}$ as a function of volume fraction $\eta$. Here $f$ is the Helmholtz free energy per volume, $\mu_{c}$ is the coexistence chemical potential, and $P_{c}$ is the coexistence pressure. This choice of representation ensures that the $\eta$-axis acts as a common tangent to the free energy.

determine the phase boundaries between the IL and SC phase. We employed Widom insertion ${ }^{47,48}$ to determine the free energy of the IL phase and Einstein integration ${ }^{49-51}$ to determine the free energy of the SC; see Appendix A for further details. For $L / D=4.0$ we found coexistence densities $\eta_{\mathrm{I}}=0.344$ and $\eta_{\mathrm{SC}}=0.385$ using a common-tangent construction, see Fig. 5(b).

By determining the EOSs for several conveniently chosen $L / D$ and performing free-energy calculations, as well as studying order parameters and their associated susceptibilities, we were able to establish the full phase diagram of hard octapods in a planar quasi-2D system, see Fig. 6. Note that in addition to the IL, RC, SC, and BSC phase, we also found a hexagonal plastic crystal (rotator) phase (HR). In the following we will classify the nature of the phases and phase transitions that we found.

We found a density jump between the IL and SC phase at coexistence, which is indicative of a first-order phase transition. To confirm the IL-SC phase coexistence, we performed $N V T$ simulations for octapods with $L / D=4.0$, which are similar to the direct-coexistence simulations of Ref. 52. We prepared a SC and an IL phase with an equal number of particles using the respective coexistence densities $\eta_{\mathrm{I}}=0.344$ and $\eta_{\mathrm{SC}}=0.385$ that we had determined using our free-energy calculations. We brought these phases into contact and equilibrated the system using $3 \times 10^{6}$ Monte Carlo cycles, where one cycle is understood to be one translation or rotation move per particle. Figure 7 shows the initial and final configurations of the coexistence $N V T$ simulations. There is a clear boundary between the SC and isotropic phase. By preparing equivalent systems with non-coexistence densities, we could observe the melting of the crystal phase into the liquid.

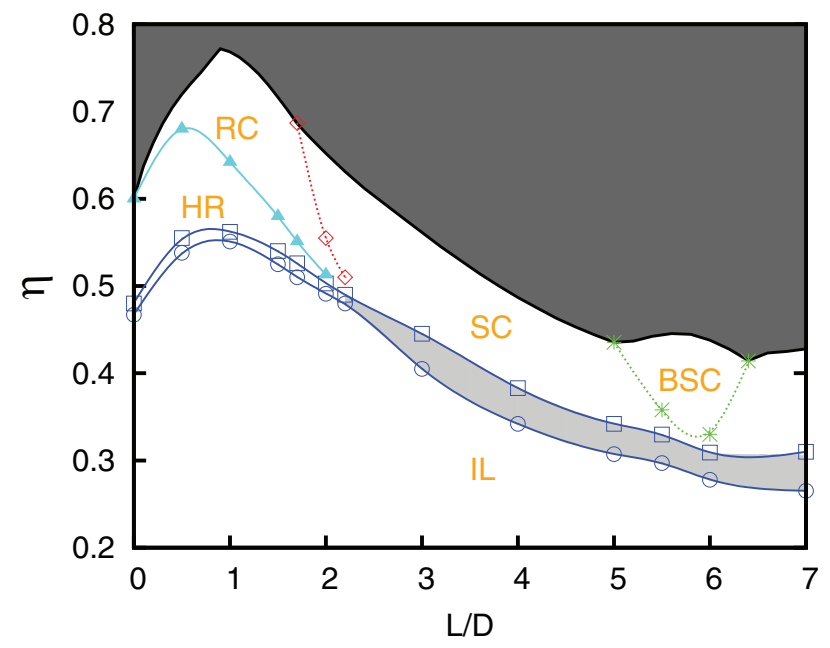

FIG. 6. The phase diagram for hard spherocylinder-based octapods in a planar quasi-2D system. We show the volume fraction $\eta$ as a function of the length-to-diameter ratio $L / D$. The light-grey area indicates the coexistence region and the dark-grey area indicates the forbidden region above the maximum packing fraction (thick black line) of the densest-known crystal. "SC" denotes the stable square-lattice crystal, "BSC" denotes the binary-lattice square crystal, "RC" denotes the rhombic crystal, and "HR" denotes the stable hexagonal plastic crystal (rotator) phase. The blue circles indicate the isotropic-liquid (IL) phase-coexistence volume fraction, the blue squares the $\mathrm{HR}$ and $\mathrm{SC}$ coexistence volume fractions. The solid blue lines are a guide to the eye. The SC-BSC transition indicated by green stars and thin dotted lines, the RC-HR transition is indicated by light-blue triangles and thin solid line, and the RC-SC transition is indicated by red squares and thin dotted line.

This melting occurs at the boundary between the SC and IL phase, see the integral multimedia movie in the supplemental material. ${ }^{53}$

A BSC was observed at high density for octapods with $L / D \in[5.1,6.3]$. The stability of this phase at high pressures

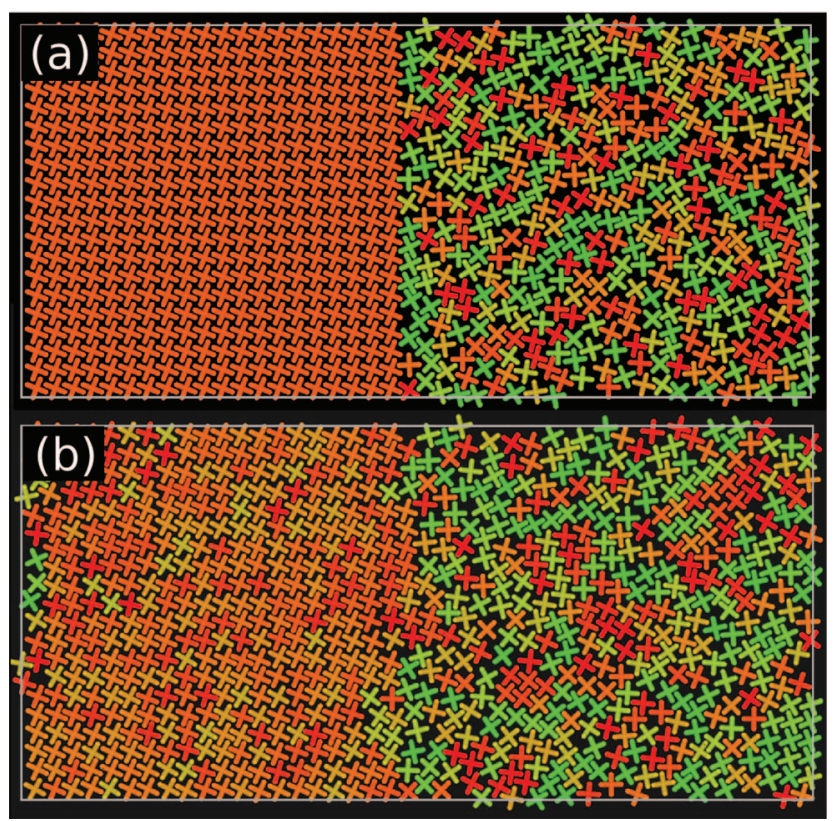

FIG. 7. Snapshots of an $N V T$ Monte Carlo simulation for which there is phase coexistence between the isotropic (right) and square crystal (left) phase for octapods with $L / D=4.0$. (a) Initial configuration for the coexistence simulation with $\eta_{\mathrm{I}}=0.344$ and $\eta_{\mathrm{SC}}=0.385$ and (b) final configuration for the coexistence simulation after $3 \times 10^{6}$ Monte Carlo cycles. The color indicates the orientation of the octapods. 

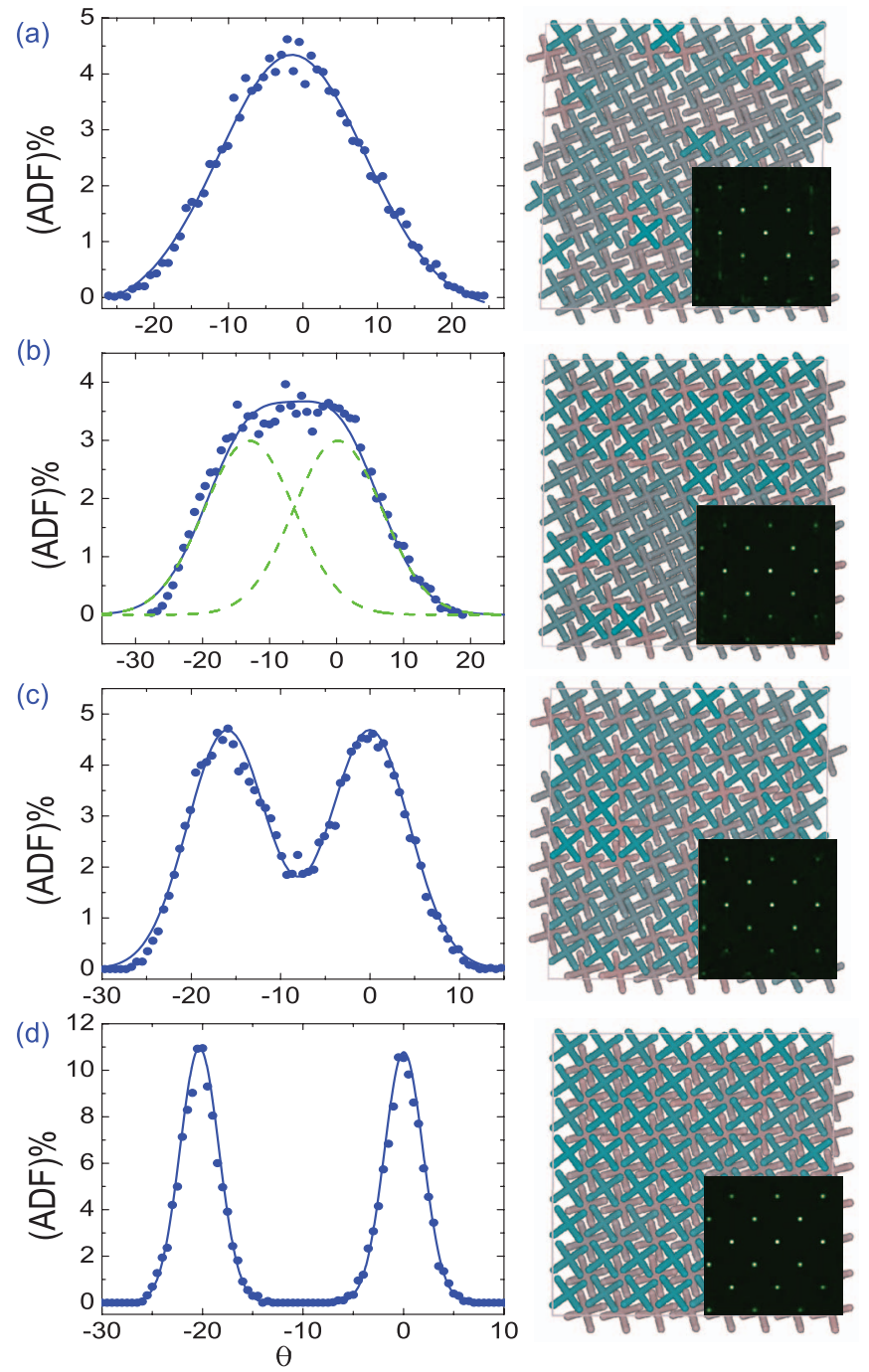

FIG. 8. (Left column) The angle distribution function (ADF) of the difference in orientation $\theta$ (in degrees) between neighboring octapods with $L / D$ $=6.0$ for several values of the reduced pressure $P^{*}=P D^{2} / k_{B} T$. (Right column) We also show snapshots and structure factors based on the centres of the particles (insets) for the systems to illustrate their state: (a) $P^{*}=0.230$, (b) $P^{*}$ $=0.260$, (c) $P^{*}=0.280$, and (d) $P^{*}=0.450$. The blue dots show measured values for the distribution and the blue lines show a single or double-Gaussian fit to the simulation results. The dashed green lines in (b) give the distribution function obtained by a double-Gaussian fit.

was confirmed by starting a simulation in a SC arrangement and allowing the system to evolve. In all cases the SC rearranged to form a BSC. By decreasing the pressure/density the BSC deformed into the SC. The EOS appeared continuous and we therefore concluded that this solid-solid phase transition is continuous or very weakly first-order. This was further confirmed using free-energy calculations, which showed a continuous free energy within the error bar. Moreover, crystallographic analysis shows that both the SC and the BSC phase belong to the same space (wall-paper) group $p 4$. Note that if only the centres of mass are considered the space group for both phases would be $p 4 m$. Remarkably, only the orientation of the particles changed during the transition, not the lattice itself. The transition point between the SC and BSC could be identified using the distribution of the orientation difference between neighboring particles, see Fig. 8, which shows four of these distributions for several pressures for oc-

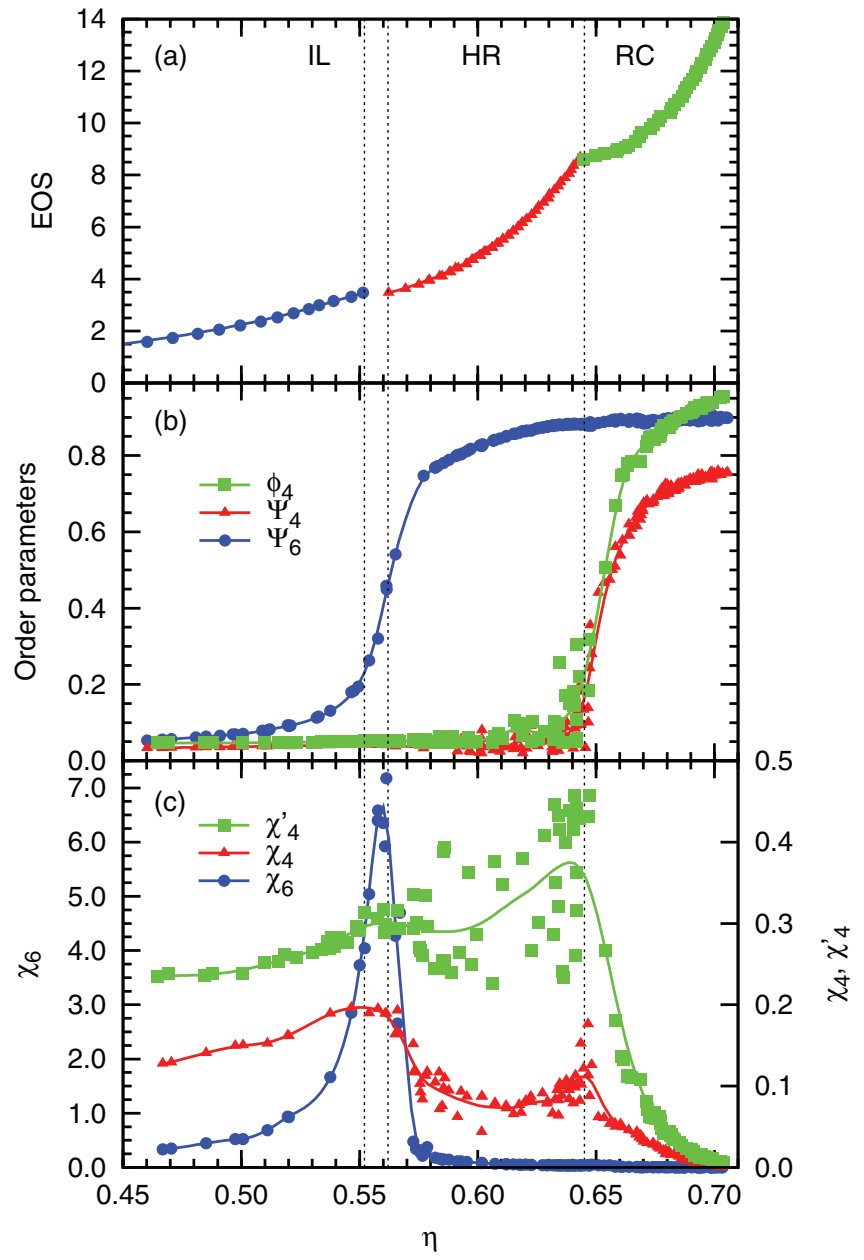

FIG. 9. Equation of state (EOS) and the packing-fraction $(\eta)$ dependence of several order parameters $\left(\phi_{4}, \Psi_{4}, \Psi_{6}\right)$ and their susceptibilities for octapods with $L / D=1.0$. (a) The EOS for this system, i.e., reduced pressure $P^{*}$ $=P D^{2} / k_{B} T$ as a function of $\eta$. The isotropic-liquid (IL) phase is denoted by blue circles, the hexagonal-rotator phase (HR) is denoted by red triangles, and rhombic crystal (RC) is denoted by green squares. (b) The global 6-fold bond orientational order parameter $\Psi_{6}$ (blue circles), the global 4-fold bond orientational order parameter $\Psi_{4}$ (red triangles), and the global orientational order parameter $\phi_{4}$ (green squares). (c) The susceptibility of the 6-fold bond orientational order parameter $\chi_{6}$ (blue circles), the susceptibility of the 4fold bond orientational order parameter $\chi_{4}$ (red triangles), and the susceptibility of the global orientational order parameter $\chi_{4}^{\prime}$ (green squares). We have added dashed vertical lines to indicate the location of the phase boundaries. The solid lines in (b) and (c) are guides to the eye.

tapods with $L / D=6.0$. In the SC phase (Fig. 8(a)) we obtained a single-peak Gaussian distribution because all particles have nearly the same orientation. For the BSC phase the distribution has a double-peak Gaussian nature because there are two different orientations, one for each sublattice [Figs. 8(c) and 8(d)]. Near the BSC to SC phase transition the double-peak merged into a single plateau (Fig. 8(b)). We also calculated the structure factor based on the centre-of-mass position of each particle in Fig. 8 (right column), which showed that the SC structure does not change during the SC-BSC phase transition.

The RC was found to be the stable phase at high pressures for octapods with $L / D \in[0.0,1.7]$; however, at lower pressures the phase appeared to persist for $L / D \in[0.0,2.2]$. Figures 9 and 10 show the pressure, several of the 
order parameters, and the susceptibilities, which were used to establish the location of the phase transitions, as a function of volume fraction. For $L / D=1.0$ the $\mathrm{RC}$ transitions into a HR phase upon lowering the pressure, the EOS is continuous at the solid-solid phase transition point (Fig. 9(a)). However, the susceptibility of 4-fold bond orientational order parameter $\chi_{4}$ shows divergent behavior, allowing us to locate the phase transition. We also calculated the 4-fold orientational order parameter $\phi_{4}$ and its susceptibility $\chi_{4}^{\prime}$. The order parameter $\phi_{4}$ drops from $\phi_{4}$ $>0.8$ to nearly 0 in the RC-HR transition, as is to be expected, since the HR phase has no inherent orientational order. There is also a peak in the corresponding susceptibility $\chi_{4}^{\prime}$ for this transition, since the susceptibility should diverge at the transition. We believe the RC-HR ( $p m m$ to $p 6 m$ ) phase transition to be continuous, because in Ref. 38 a similar RC-HR transition in a $2 \mathrm{D}$ system of hard squares was found to be continuous and, as we mentioned earlier, there are strong analogies between both systems for $L / D<2.0$. However, within the present level of accuracy of our simulations we were not able to verify this property. The HR phase melted into an isotropic (liquid) phase by further lowering the pressure. We demonstrated that this phase transition is first order. The coexistence volume fractions were located by free-energy calculations. Moreover, the susceptibility of 6-fold bond orientational order $\chi_{6}$ diverges at the coexistence density.

For octapods with $L / D=2.0$ the densest structure is a SC. The SC melted into a $\mathrm{RC}$ at lower pressures, which melted into a HR phase and eventually into the IL phase. At the SC$\mathrm{RC}$ phase transition point, the 6-fold bond orientational order parameter $\Psi_{6}$ decreased from 0.9 to nearly 0.0 because there are no 6-fold bonds in the SC. We used the susceptibility of the global 4-fold (Fig. 10(c)) and 6-fold (Fig. 10(b)) orientational order parameters $\left(\Psi_{4}\right.$ and $\left.\Psi_{6}\right)$, respectively, to determine the location of the other phase transitions. Because we did not observe a density jump (Fig. 10(a)), this phase transition is likely continuous, possibly weak first order, since there appears to be a discontinuity in $\partial P / \partial \eta$. However, it is at this time impossible to determine with certainty what type this transition is, because of the numerical limitations of our algorithm and the finite-size effects that play a role in the regime we can access.

For $L / D=0.0$, the octapod model is the same as a hard sphere. Moreover, due to the confinement in the planar quasi2D geometry the system can be mapped onto one consisting of monodisperse hard disks. The crystal phase is the hexagonal (rotator) phase. For hard disks, there is a possible hexatic phase between the crystal and liquid phase. ${ }^{42}$ In our simulations, we also found indications of such a hexatic phase, but as we were constrained by the system size, we were not able to draw definite conclusions that such a phase is indeed present. By analysing the 6-fold bond orientational correlation function $g_{6}(r)$ for various densities we attempted to characterize the IL-HR phase transition for $L / D=2.0$, see Fig. 11 . We found that the bond orientational correlation functions exhibited an algebraic decay with slope near to $1 / 4$ for $\eta \approx 0.5$, which hints at a KT-type transition as predicted by KTHNY theory. ${ }^{39-41}$ However, for the relatively small system sizes we considered, it is not possible to exclude that there is

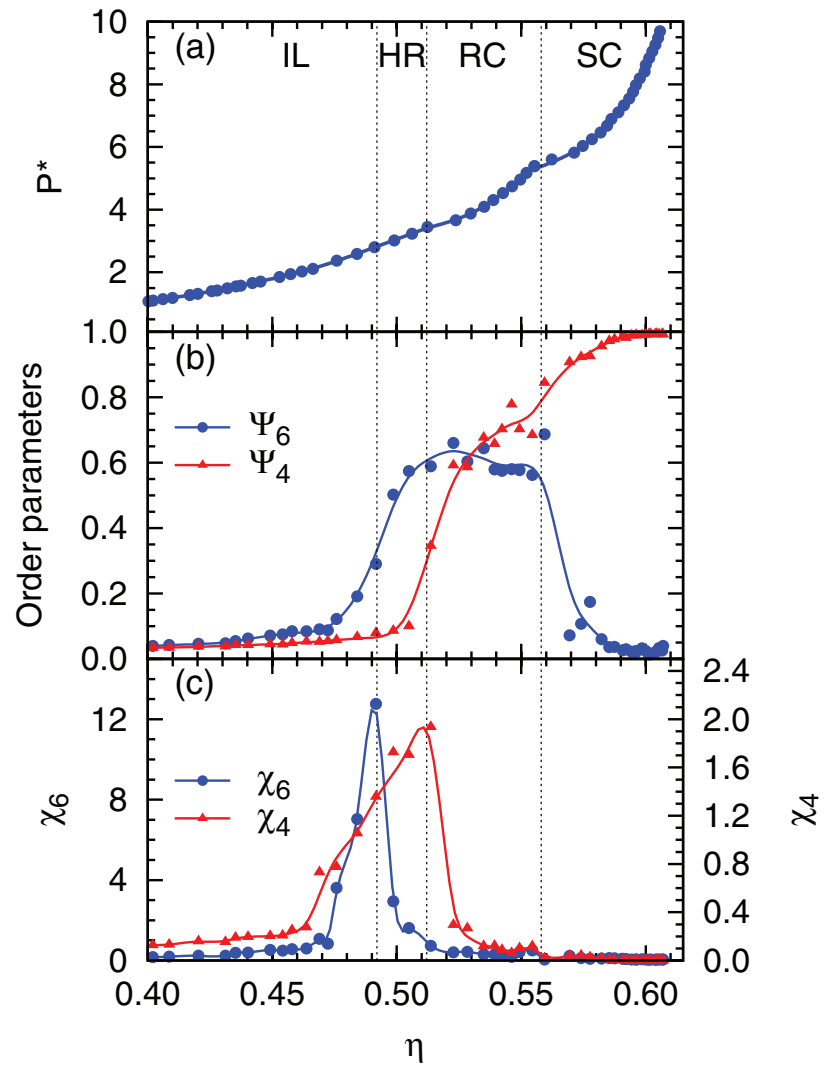

FIG. 10. The packing-fraction $(\eta)$ dependence of several order parameters and their susceptibilities for octapods with $L / D=2.0$. (a) The EOS for this system with $P^{*}=P D^{2} / k_{B} T$ the reduced pressure. We labelled the square crystal phase using "SC," the rhombic crystal phase using "RC," the hexagonal rotator phase using "HR," and the fluid phase using "IL." (b) The global 6fold and 4-fold bond orientational order parameter $\Psi_{6}$ (blue circles) and $\Psi_{4}$ (red triangles), respectively. (c) The susceptibility $\chi_{6}$ (blue circles) and $\chi_{4}$ (red triangles) as a function of the packing fraction. The packing fractions corresponding to peaks in the susceptibilities (dashed vertical lines) give the location of the phase transitions. The solid lines in (b) and (c) are guides to the eye.

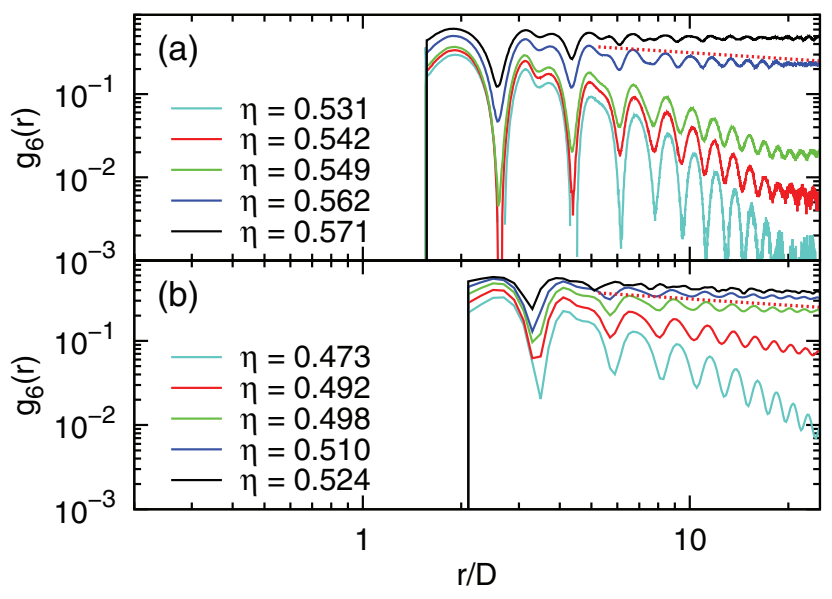

FIG. 11. The 6-fold bond orientational correlation function $g_{6}$ as a function of the radial distance $r$ from the centre of an octapod for several packing fractions $\eta$ and $L / D=2$ (a) and $L / D=1$ (b), respectively. Results for different values of $\eta$ are indicated by different colors. The thick red dashed line has a slope of $1 / 4$ and corresponds to the power-law decay predicted by KTHNY theory. 
coexistence via an intermediate hexatic phase. For $L / D=1.0$ free-energy calculations showed that the HR-IL phase transition is first-order. However, due to finite size effects, the $N V T$ simulations for this system also showed that $g_{6}(r)$ decays with a power law and an algebraic index close to $1 / 4$ in the coexistence phase. Therefore, further studies are required to establish whether KT-transitions occur in the region $L / D \in[0$, 2].

In conclusion, we mapped out the full phase diagram of hard octapods in a planar quasi-2D system using free-energy calculations and an analysis based on several order parameters and their corresponding susceptibilities. We found a firstorder phase transition from a fluid to a HR or SC phase, depending on the aspect ratio. Additionally, we find a weak firstorder or continuous HR-RC phase transition for $L / D<2.2$. More surprisingly, we find three different phase transitions, i.e., fluid-HR, HR-RC, and a RC-SC, with increasing packing fraction for hard octapods with an aspect ratio $L / D \simeq 2$

\section{CONCLUSION AND OUTLOOK}

We examined by simulations the experimental observations of the formation of crystalline monolayers consisting of octapod-shaped nanocrystals, which arranged in a square-lattice crystal, by drop-casting a suspension of octapods on a substrate and allowing the solvent to evaporate. In the experiments square-lattice crystals were found for a pod length-to-diameter ratio $(L / D)$ of 4.8 , whereas for $L / D \approx 5.9$ binary-lattice square crystals were observed in the samples in addition to square-lattice crystals. These experimental results could be explained using Monte Carlo simulations, in which we described the octapod-shaped nanocrystals by a hard-particle model consisting of four interpenetrating spherocylinders. Our model is completely determined by the length-to-diameter ratio $(L / D)$ of the spherocylinders, with $L$ the length and $D$ the diameter. By constraining these octapod models to move in a planar quasi-2D geometry, namely that of the monolayers observed in the experiments, the formation of crystals could be studied. We determined the high-density crystal structures using floppy-box Monte Carlo simulations ${ }^{16,44,45}$ and subsequently established the equations of state (EOSs) for various values of $L / D$. This enabled us to construct the full phase diagram as a function of $L / D$ and establish the nature of the various phase transitions, using free-energy calculations, as well as global bond-orientational order parameters and their associated susceptibilities.

In addition to the isotropic liquid (IL) phase, we found a variety of crystal phases: a rhombic crystal (RC), a squarelattice crystal (SC), a binary-lattice square crystal (BSC), and a hexagonal plastic-crystal (rotator) phase (HR). Our results appear to be consistent with the experimental observations, ${ }^{8}$ that is, we found a high-density SC phase for $L / D \in[1.8$, 5.0] $\cup[6.3,8.0]$ and a BSC phase for $L / D \in[5.1,6.2]$. For $L / D \in[0.0,1.7]$ we observed that the RC melted to an isotropic phase, via a hexagonal plastic-crystal (rotator) phase (HR). In the region $L / D \in[1.7,2.0]$ there appears to be a three-step phase transition; by reducing the pressure the SC melts into the RC, which subsequently melts into the HR phase, and finally the HR phase melts into the isotropic liquid phase. In this region, for specific values of $L / D$ the algebraic decay of the bond-orientational correlation function in the HR phase indicates the possibility of a KT-type transition to the IL phase. We confirmed that for $L / D>2.0$ the SC melted into an IL phase via a first-order phase transition using free-energy calculations and direct (coexistence) isothermalisochoric $(N V T)$ simulations. In the range $L / D \in[5.1,6.2]$ the BSC is stable at high pressures and melts into the SC for lower pressures, which subsequently melts into the isotropic phase via a first-order phase transition upon lowering the pressure further. A continuous phase transition between the BSC and $\mathrm{SC}$ was observed, for which only the relative orientations of the particles in the BSC changed and the crystal lattice remained unaffected.

The results gained by our simulation studies show that $\mathrm{RC}, \mathrm{SC}$, and BSC monolayers may be prepared in future experiments by tuning the pod length-to-diameter ratio. Moreover, the present work can be used as an initial step toward a better understanding of the (out-of-equilibrium) formation of crystalline monolayers of branched nanocrystals. These insights might help the optimization of the experimental conditions to achieve large and defect-free crystalline monolayers. These larger 2D assemblies might be of use in device applications. The techniques introduced and used in this paper are also of significant interest to future simulation studies, since they can also be applied to investigate the behavior of other types of (nonconvex) nanoparticles on substrates, or air-liquid as well as liquid-liquid interfaces, e.g., binary nanoparticle superlattices, ${ }^{54,55}$ truncated cubes,${ }^{56}$ tetrapods, and nanostars.

\section{ACKNOWLEDGMENTS}

It is a pleasure to thank Dr. G. Bertoni, Dr. R. Brescia, A. P. Gantapara, Dr. R. Ni, Dr. F. Smallenburg, Dr. D. Ashton, and Professor R. van Roij for useful discussions. We would also like to thank K. Miszta for help with the synthesis of octapods. M.D. acknowledges financial support by a "Nederlandse Organisatie voor Wetenschappelijk Onderzoek" (NWO) Vici Grant, and J.d.G. by the Utrecht University High Potential Programme. L.M. acknowledges financial support from the European Union through the FP7 starting ERC grant NANO-ARCH (Contract No. 240111).

\section{APPENDIX A: FREE-ENERGY CALCULATIONS}

In this appendix we explain in detail the way in which we determined the free energy of the various (plastic) crystal phases using Einstein integration. ${ }^{49-51}$ In the Einstein integration method the positions of the particles, as well as their orientations, are coupled to the respective positions and orientations of the particles in the Einstein crystal by Hookian springs. These springs are described by the external potential

$$
\beta U(\lambda)=\lambda \sum_{i=1}^{N}\left[\frac{\left(\boldsymbol{r}_{i}-\boldsymbol{r}_{i}^{0}\right)^{2}}{D^{2}}+\left(1-\cos 4 \psi_{i}\right)\right],
$$

where $N$ is the number of particles, $\beta=1 / k_{B} T$ is the inverse thermal energy, $\lambda$ is the coupling constant, $\boldsymbol{r}_{i}$ is the position 


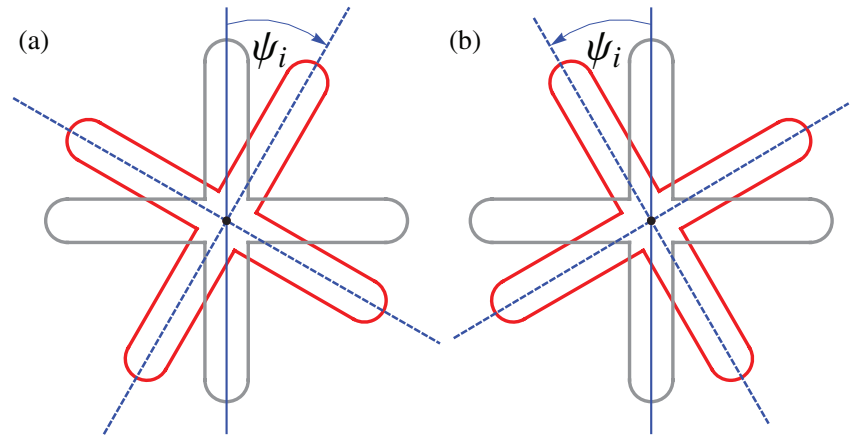

FIG. 12. Illustration of the definition of the minimum angle $\psi_{i} \in[-\pi / 4$, $\pi / 4]$. The grey octapod indicates the Einstein-crystal reference frame and the red octapod shows the orientation of the octapod of interest. The blue lines indicate the cylinder centres. We consider two clockwise rotations of the octapod, a small one by less than $\pi / 4$ (a) and a larger one by slightly more than $\pi / 4$ (b). For the former we obtain a positive $\psi_{i}<\pi / 4$ value, whereas for the latter we use the symmetry to map the rotation on a negative $\psi_{i}>-\pi / 4$ value.

of the $i$ th particle's centre, $\boldsymbol{r}_{i}^{0}$ is the position of the lattice site associated with the $i$ th particle's centre in the Einstein crystal, and $\psi_{i}$ is the minimum angle that gives the difference in orientation of the four spherocylinders comprising the octapod model and the orientation of this model in the Einstein crystal, see Fig 12.

It is possible to use different coupling constants for the positional and orientational parts, but we chose not to do this here, because this may introduce artefacts. The coefficient 4 in the cos term accounts for the fourfold symmetry of octapods. In the BSC phase, we required different orientational springs for the two sublattices. When the spring constant $\lambda$ is sufficiently high, the particle positions and orientations are bound so tightly to the lattice sites and particle orientations in the Einstein crystal that the particles effectively no longer interact. The system therefore behaves as an noninteracting Einstein crystal. We denote the cut-off value for which the system is effectively noninteracting by $\lambda_{m}$. By decreasing the spring constant from this noninteracting state to the state of interest it is possible to determine the free energy of that state by thermodynamic integration.

The free energy of the quasi-2D noninteracting Einstein crystal with a centre-of-mass correction, from which we integrate to the desired state, is given by

$$
\frac{\beta F_{\text {Ein }}}{N}=-\frac{N-1}{N} \log \frac{\pi}{\lambda_{m}}-\frac{\log A}{N}+\frac{\beta F_{\text {ori }}}{N},
$$

where $\log$ is the natural $\operatorname{logarithm}, A$ is the area of the $x y$ plane enclosed by the simulation box, and $F_{\text {ori }}$ is the orientational free energy of the noninteracting Einstein crystal, which may be written as

$$
\frac{\beta F_{\text {ori }}}{N}=-\log \left\{\frac{1}{2 \pi} \int_{0}^{2 \pi} \exp \left[-\lambda_{m}\left(1-\cos 4 \psi_{i}\right)\right] \mathrm{d} \theta\right\},
$$

where the integral is taken over all possible orientations of a single octapod. It is easily verified that $\cos 4 \psi_{i}=\cos \theta$ for this integration, where $\psi_{i}$ is the minimum angle as before and the factor 4 indicates that the configurations are 4-fold degenerate when $\theta$ changed from 0 to $2 \pi$.

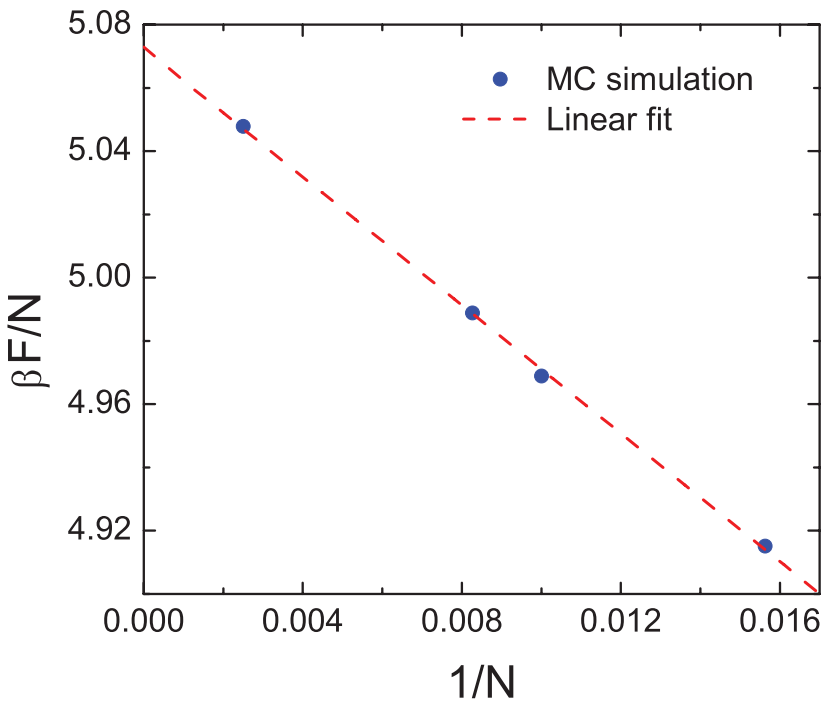

FIG. 13. Finite size scaling for the free energy per particle $\beta F / N$ obtained by Einstein integration for a system of hard octapods with a packing fraction $\eta=0.40$ and a pod length-to-diameter ratio of $L / D=4.0$. The blue dots show the results of Monte Carlo simulations for $N=64,100,121$, and 400 particles. The dashed red line shows a linear fit to the data, by which it is possible to determine the free energy of this phase. In the limit $N \rightarrow \infty$ we obtain $\beta F / N=5.073$.

We used the above equations to determine the free energy of a system at fixed $L / D$ and packing fraction $\eta$. In our simulations, we found that a value of $\lambda_{m}=3,000$ sufficed to obtain a noninteracting system. To determine the free energy of the bulk phase we used finite-size scaling, ${ }^{57}$ by which we extrapolated our results from finite $N$ to $N \rightarrow \infty$. This procedure is illustrated in Fig. 13.

\section{APPENDIX B: ORDER PARAMETERS AND CORRELATION FUNCTIONS}

In this appendix, we briefly introduce the various order parameters and correlation functions we used to distinguish between different phases and by which we were able to locate the packing fractions and pressures at which the (continuous) phase transitions occurred.

- The global $n$-fold bond orientational order parameter: ${ }^{25,40}$

$$
\Psi_{n}=\left\langle\frac{1}{N} \sum_{j=1}^{N} \frac{1}{N_{b}} \sum_{k=1}^{N_{b}} \exp \left(i n \theta_{j k}\right)\right\rangle,
$$

where $i$ is the imaginary unit, $N$ is the number of particles, $N_{b}$ is the number of nearest neighbors, $\theta_{j k}$ is the angle between the bond of two neighboring particles $(j$ and $k$ ) and an arbitrary reference axis, and $\langle\cdot\rangle$ indicates ensemble averaging. The nearest neighbors are defined by using a Voronoi construction. In our studies we used the 4-fold and 6-fold bond orientational order parameter $\Psi_{4}$ and $\Psi_{6}$, respectively. This choice is based on the ability of these parameters to determine the level of square and hexagonal order, respectively, present in the system. This makes the pa- 
rameters suited to study the hexagonal rotator phaserhombic crystal phase (HR-RC) transition.

- The $n$th order bond orientational correlation function is defined as

$$
g_{n}(r)=\left\langle\phi_{n}\left(r_{0}\right) \phi_{n}\left(r_{0}+r\right)\right\rangle,
$$

where $\phi_{n}(r)=N_{b}^{-1} \sum_{k=1}^{N_{b}} \exp \left(i n \theta_{j k}(r)\right)$ is the $n$th order local orientational order parameter, $r$ is the radial distance from the central octapod, and $r_{0}$ is position of the central octapod. This parameter quantifies the extent to which orientational order is maintained in the direction of the nearest neighbor bonds in the crystal. The nature of the decay in this parameter can therefore give some insight into the possible presence of a Hexatic phase, which is characterized by power-law decay of the 6-fold bond orientational correlation function (with power $1 / 4$ as predicted by KTHNY theory), rather than exponential decay.

- The global 4-fold orientational order parameter is given by

$$
\phi_{4}=\left\langle\frac{1}{N} \sum_{k=1}^{N} \exp \left(i 4 \theta_{k}\right)\right\rangle,
$$

where $\theta_{k}$ is the angle any pod of the octapod makes with an arbitrary reference axis. This parameter measures the level of orientational order in the system, i.e., to what extend the octapods are aligned on average. It can therefore be used to differentiate between states with high order, such as crystalline and nematic/tetratic phases, and states with orientational disorder, such as isotropic liquids and rotator phases.

- The susceptibility of the $n$-fold bond order parameter is determined by calculating the fluctuations of the bond order parameters

$$
\chi_{n}=\left\langle\Psi_{n}^{2}\right\rangle-\left\langle\Psi_{n}\right\rangle^{2} .
$$

The susceptibility of the orientational order parameter is defined as

$$
\chi_{4}^{\prime}=\left\langle\phi_{4}^{2}\right\rangle-\left\langle\phi_{4}\right\rangle^{2} .
$$

Effectively, the susceptibilities give information on the location of the phase boundaries in our system, since the susceptibility is divergent near such a boundary.

To determine these order parameters using Monte Carlo (MC) simulations, we typically used the following strategy. First we performed $10^{6} \mathrm{MC}$ steps in the $N P T$ ensemble using variable-box-shape MC simulations with $N=400$ octapods. The final configuration was then used in a $\mathrm{MC}$ simulation in the NVT ensemble, where we used $2 \times 10^{6} \mathrm{MC}$ steps for equilibration and $5 \times 10^{5} \mathrm{MC}$ steps for production.

\section{APPENDIX C: FREE-VOLUME THEORY}

We compared our Monte Carlo simulation results for the EOS of the SC phases with the results of a free-volume (or rather area) theory based on a cell-model approach. ${ }^{58}$ In this cell model, it is assumed that the central particle is caged by its four neighbors in an expanded SC geometry and that these

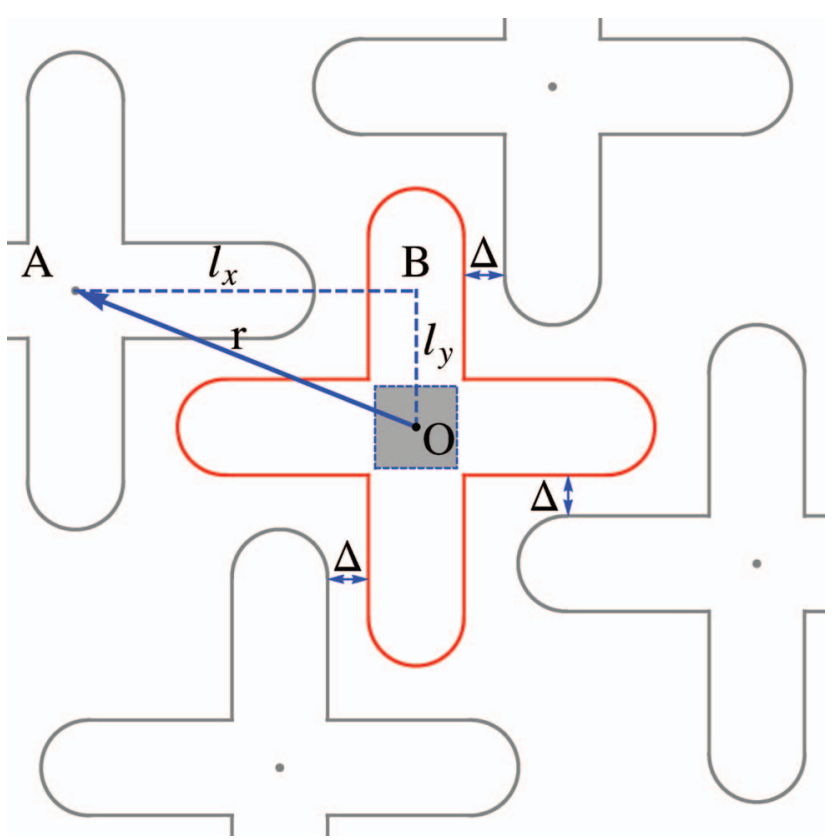

FIG. 14. Top view of the cell model for a central octapod (red) that is only allowed to translate in the $x y$-plane, surrounded by four neighboring positionally and orientationally fixed octapods (grey), which are arranged according to the square-lattice crystal (SC) structure. The SC structure has been expanded uniformly to achieve a desired volume fraction $\eta$. The lengthto-diameter ratio $L / D=4.0$ in this case. The centre-to-centre vector between the central octapod and its top-left neighbor is given by $\mathbf{r} \equiv O A$ $=\sqrt{O B^{2}+A B^{2}}$, with $A B=l_{x}$ and $O B=l_{y}$. The parameter $\triangle$ gives the size of the gap between neighboring particles. The grey square identifies the area in which the centre of the central octapod is free to move, i.e., its free area (volume).

neighbors are positionally and orientationally fixed, i.e., we use a mean-field approximation. In order to explain how the free area can be calculated we start from a simplified situation, before we consider the full cell model. Let us first consider the situation in which the central octapod can only translate in the $x y$-plane; rotations are not allowed. Later we will extend this result to the situation where rotations of the central octapod around the $z$-axis are also allowed.

Figure 14 shows the top view of the cell model for a central octapod which is only allowed to translate in the $x y$-plane. In the close-packed SC configuration the centre-to-centre distance between two octapods is $\mathbf{r}^{c p}$. It proves convenient to decompose $\mathbf{r}^{c p}$ into its $x$ - and $y$-components, which we denote by $r_{x}^{c p}$ and $r_{y}^{c p}$, respectively. From the dense-packed configuration, it is obvious that $r_{y}^{c p}$ is equal to $D$. However, $r_{x}^{c p}$ depends on the length of octapods. The analytical expression for $r_{x}^{c p}$ is given by

$$
\frac{r_{x}^{c p}}{D}= \begin{cases}\frac{\sqrt{3} L^{*}+\sqrt{12-2 \sqrt{6} L^{*}-L^{* 2}}}{3 \sqrt{2}} & \frac{\sqrt{6}}{2} \leq L^{*} \leq \frac{5 \sqrt{6}}{2} \\ \frac{L^{*}+\sqrt{\left(2 \sqrt{6}-L^{*}\right) L^{*}}}{\sqrt{6}} & L^{*} \leq \frac{\sqrt{6}}{2} \\ 3 & \frac{5 \sqrt{6}}{2} \leq L^{*} \leq 8,\end{cases}
$$

where $L^{*}=L / D$ is the reduced length of octapods, and the upper bound of $L^{*}=8$ is the point up to which we could numerically verify our result. 
In the cell model, we assume that the SC structure does not change with the volume fraction. We therefore expand the SC configuration uniformly to achieve the desired value of $\eta$. This expansion can also be expressed in terms of the parameter $\Delta$, which gives the size of the gap between neighboring particles. In the expanded SC-configuration the centreto-centre distance vector is denoted by $\mathbf{r}$, and its $x$ - and $y$ components are denoted by $l_{x}$ and $l_{y}$, respectively. For the noninterlocking SC phase, i.e., $L^{*}<5$, the centre-to-centre distance between two octapods can be expressed in terms of $\mathbf{r}^{c p}$ and $\Delta$ as

$$
\begin{aligned}
r^{2} & =l_{x}^{2}+l_{y}^{2}, \\
& =2 \Delta^{2}+\left(r^{c p}\right)^{2}+2 \Delta\left(r_{x}^{c p}+r_{y}^{c p}\right) .
\end{aligned}
$$

The area $A_{f}$ in which the octapod is free to move (the grey area in Fig. 14) is given by $A_{f}=4 \Delta^{2}$. This area can be determined by solving Eq. (C2) for $\Delta$ and the expression reads

$$
\begin{aligned}
A_{f} & =4 \Delta^{2}, \\
& =2 r_{c}^{2}\left[\frac{r^{2}}{\left(r^{c p}\right)^{2}}+C_{1}-C_{2} \sqrt{2 \frac{r^{2}}{\left(r^{c p}\right)^{2}}-1+C_{1}}\right],
\end{aligned}
$$

with

$$
\begin{aligned}
& C_{1}=2 \frac{r_{x}^{c p} r_{y}^{c p}}{\left(r^{c p}\right)^{2}}, \\
& C_{2}=\frac{r_{x}^{c p}+r_{y}^{c p}}{r^{c p}} .
\end{aligned}
$$

Since the centre-to-centre distance vector between two neighboring octapods is also the lattice vector of the crystal structure in the SC phase, the volume fraction $\eta$ of the expanded SC configuration can be written as $V_{p} /\left(h r^{2}\right)$, where $V_{p}$ is the volume of an octapod. In the dense-packed configuration $\eta_{c}=V_{p} /\left(h\left(r^{c p}\right)^{2}\right)$. Therefore, we may write

$$
\frac{r^{2}}{\left(r^{c p}\right)^{2}}=\frac{\eta_{c}}{\eta}
$$

Using the relation in Eq. (C4), the free area $A_{f}$ is rewritten as

$$
A_{f}=2\left(r^{c p}\right)^{2}\left[\frac{\eta_{c}}{\eta}+C_{1}-C_{2} \sqrt{2 \frac{\eta_{c}}{\eta}-1+C_{1}}\right] .
$$

The partition function for the $2 \mathrm{D}$ system in the free-area (mean-field) approximation takes the form

$$
Q_{t}=\frac{A_{f}^{N}}{\Lambda^{2 N}},
$$

where $\Lambda$ is the De Broglie wavelength. The Helmholtz free energy per particle is therefore given by

$$
\begin{aligned}
f & =-\frac{k_{B} T}{N} \log Q_{t} \\
& =k_{B} T\left[2 \log \Lambda-\log A_{f}\right] .
\end{aligned}
$$

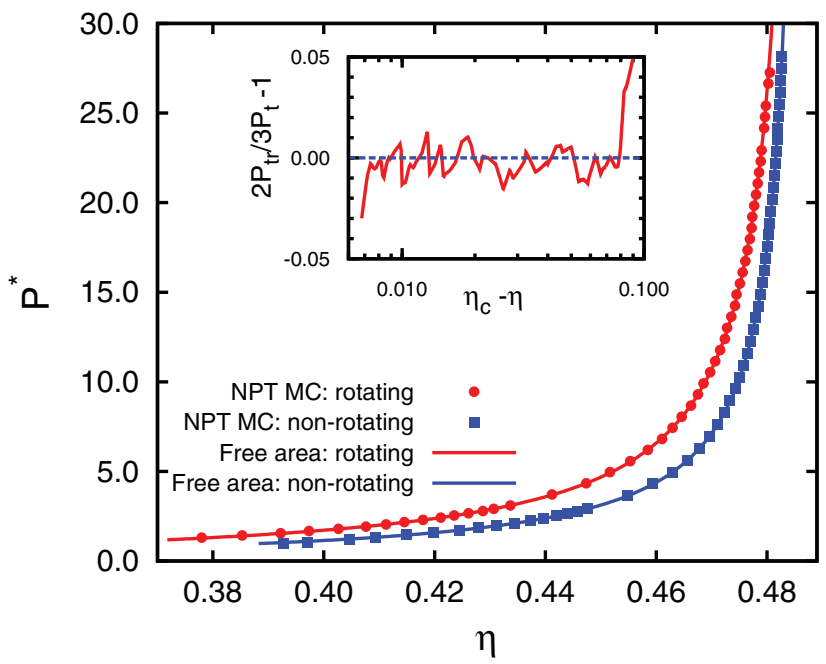

FIG. 15. The reduced pressure $P^{*}=P A / k_{B} T$ as a function of the packing fraction $\eta$ for the rotating and non-rotating octapod free-volume model with length-to-diameter ratio $L^{*}=4.0$ that follow from our theoretical calculations and from our NPT Monte Carlo (MC) simulations, respectively. Solid lines give the free-volume theory results and the dots indicate the results from our MC simulations. The inset shows $2 P_{t r} / 3 P_{t}-1$ (red line) as a function of $\eta$, where $P_{t r}$ is the reduced pressure for the NPT Monte Carlo system, in which the octapods can rotate, and $P_{t}$ is the reduced pressure for the NPT Monte Carlo system, in which the octapods can only translate. The blue dashed line indicates the ideal situation for which $P_{t r} \equiv 3 P_{t} / 2$; the red line indicates the fractional deviation with respect to this scaling.

The equation of state is obtained by using the standard thermodynamic relations

$$
\begin{aligned}
\beta P_{t}(\eta) D^{2} & =-\beta D^{2} \frac{\partial F}{\partial A}=-\beta D^{2} \frac{\partial F}{\partial \eta} \frac{\partial \eta}{\partial A} \\
& =\frac{1-\frac{C_{2}}{\sqrt{C_{1}-1+2 \frac{M_{c}}{\eta}}}}{C_{1}+\frac{\eta_{c}}{\eta}-C_{2} \sqrt{C_{1}-1+2 \frac{\eta_{c}}{\eta}}},
\end{aligned}
$$

where $P_{t}$ is the pressure in the non-interacting SC phase according to the cell model that only allows translations of the octapods. The values of $C_{1}, C_{2}$, and $\frac{\eta_{c}}{\eta}$ can be determined from the value of $r^{c p}$ and $r_{x}^{c p}$.

We compared the results from the free-volume (area) theory to the results of our Monte Carlo simulations, see Fig. 15, which shows this comparison for non-rotating octapods with $L / D=4$.0. As can be seen from Fig. 15 the results of our free-volume theory closely follow the results of the rotationally constrained isothermal-isobaric (NPT) simulations.

Let us now consider the model in which the central particle can rotate around the $z$-axis and translate in the $x y$-plane, see Fig. 16. The angle the central octapod makes with its neighbors is indicated by $\phi$. The free area is now a function of this angle, i.e., $A_{f}(\phi)$. The partition function for the cell model that allows translations and rotations is given by

$$
\begin{aligned}
Q_{t r} & =\frac{1}{\Lambda^{2 N}}\left[\int \exp (-\beta U) d r_{x} d r_{y} d \phi\right]^{N}, \\
& =\frac{1}{\Lambda^{2 N}}\left[\int_{\phi_{m}}^{\phi_{M}} A_{f}(\phi) d \phi\right]^{N}, \\
& =\frac{1}{\Lambda^{2 N}} \Sigma^{N}
\end{aligned}
$$




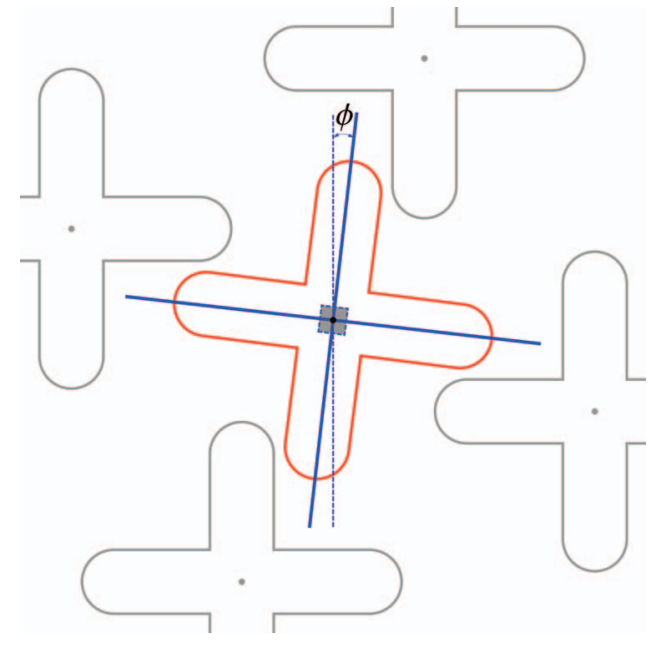

FIG. 16. A top view of the cell model, in which the central octapod (red) is allowed to rotate and translate with respect to its neighbors (black). We used a length-to-diameter ratio $L / D$ of 4.0 here. The angle which the central octapod makes with its fixed neighbors is given by $\phi$.

where $\Lambda$ is the thermal wavelength, $\beta \equiv 1 / k_{\mathrm{B}} T$ is the inverse thermal energy, $U$ is the hard-interaction potential between the central octapod and its neighbors $(U=0$ when there are no overlaps and $U=\infty$ when there are), and $\phi_{m}$ and $\phi_{M}$ are boundaries to the domain for which $A_{f}(\phi) \neq 0 . \Sigma$ is the integration of $A_{f}(\phi)$ from $\phi_{m}$ to $\phi_{M}$. When $\phi$ is $0, A_{f}(0)=A_{f}$. However, the general expression for $A_{f}(\phi)$ is nontrivial, and therefore $\Sigma$ could only be determined using Monte Carlo integration techniques. In our Monte Carlo integration we allowed the central particle to explore non-overlapping configurations to approximate both the shape and size of the free area; four-decimal precision could be obtained for $A_{f}(\phi)$. In Fig. 17, we show the numerically determined $A_{f}(\phi)$ as a function of $\phi$, from which we determined the partition function for the freely rotating octapods.

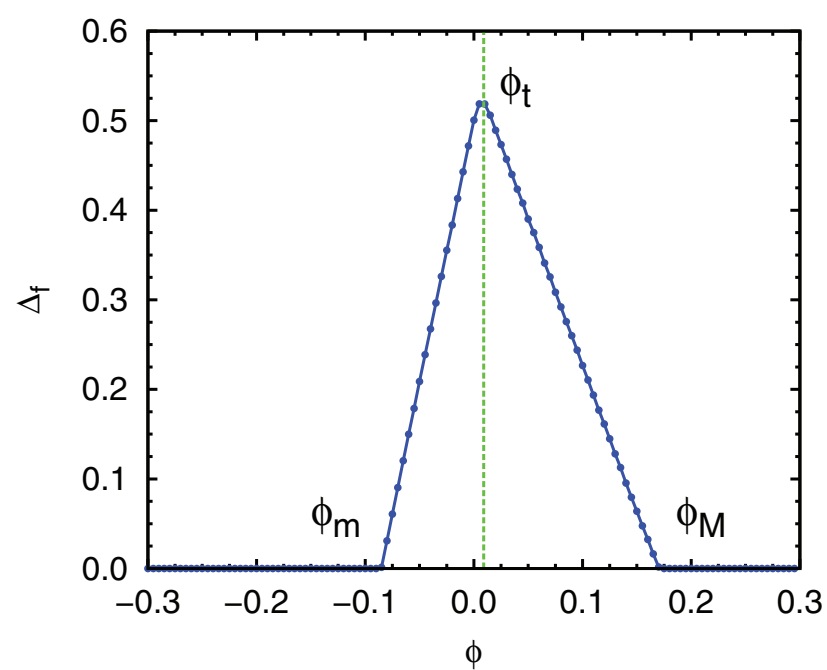

FIG. 17. The length $\Delta_{f}(\phi)=\sqrt{A_{f}(\phi)}$ of the square that delimits the free area available to the central octapod $(L / D=4)$, when it makes an angle $\phi$ with its neighbors. Note that for $\phi<\phi_{m}$ and $\phi>\phi_{M} \Delta_{f}(\phi)=0$. The value of $\phi$ for which $\Delta_{f}(\phi)$ assumes its maximum is denoted by $\phi_{t}$.
It is desirable to obtain an analytic result for $\Sigma$, the integral over $A_{f}(\phi)$, despite the difficulties in determining $A_{f}(\phi)$. As can be appreciated from the inset of Fig. 15, the difference in pressure between the NPT Monte Carlo simulation results for the system in which the octapods are allowed to rotate and the system in which only translations are allowed, is a scale factor of $3 / 2$ (when $\eta$ is fixed). We obtained a fractional deviation of this ideal scaling of less than $5 \%$ (less than $2 \%$ for most data points) in the crystal branch of the EOS, up to $\eta_{c}$ $-\eta<0.007$. We are therefore justified in forgoing a full analytic calculation of $\Sigma$ and using the approximation $\Sigma$ $\propto A_{f}^{3 / 2}$. This leads to the following free-volume expression for the EOS of the octapods that are allowed to rotate: $P_{t r}(\eta)$ $=3 P_{t}(\eta) / 2$. We have therefore obtained an analytic expression for the EOS of the octapods confined in a quasi-2D squarelattice crystal phase, which is consistent with the results of our simulations for the entire crystal branch up to a high numerical accuracy.

${ }^{1}$ L. Manna, E. C. Scher, and A. P. Alivisatos, J. Am. Chem. Soc. 122, 12700 (2000).

${ }^{2}$ Y. Sun and Y. Xia, Science 298, 2176 (2002).

${ }^{3}$ N. Zhao and L. Qi, Adv. Mater. 18, 359 (2006).

${ }^{4}$ C. Quilliet, C. Zoldesi, C. Riera, A. van Blaaderen, and A. Imhof, Eur. Phys. J. E 27, 13 (2008).

${ }^{5}$ S. Deka, K. Miszta, D. Dorfs, A. Genovese, G. Bertoni, and L. Manna, Nano Lett. 10, 3770 (2010).

${ }^{6}$ K. Miszta, J. de Graaf, G. Bertoni, D. Dorfs, R. Brescia, S. Marras, L. Ceseracciu, R. Cingolani, R. van Roij, M. Dijkstra, and L. Manna, Nature Mater. 10, 872 (2011).

${ }^{7}$ J. Henzie, M. Grünwald, A. Widmer-Cooper, P. L. Geissler, and P. Yang, Nature Mater. 11, 131 (2012).

${ }^{8}$ W. Qi, J. de Graaf, F. Qiao, S. Marras, L. Manna, and M. Dijkstra, Nano Lett. 12, 5299 (2012).

${ }^{9}$ J. S. Yin and Z. L. Wang, Phys. Rev. Lett. 79, 2570 (1997).

${ }^{10}$ H. Maeda and Y. Maeda, Phys. Rev. Lett. 90, 018303 (2003).

${ }^{11}$ M. Marechal, R. J. Kortschot, A. F. Demirörs, A. Imhof, and M. Dijkstra, Nano Lett. 10, 1907 (2010).

${ }^{12}$ A. Haji-Akbari, M. Engel, A. S. Keys, X. Zheng, R. G. Petschek, P. PalffyMuhoray, and S. C. Glotzer, Nature (London) 462, 773 (2009).

${ }^{13}$ S. Torquato and Y. Jiao, Nature (London) 460, 876 (2009).

${ }^{14}$ D. W. Sinkovits and E. Luijten, Nano Lett. 12, 1743 (2012).

${ }^{15}$ M. Marechal and M. Dijkstra, Phys. Rev. E 82, 031405 (2010).

${ }^{16}$ J. de Graaf, R. van Roij, and M. Dijkstra, Phys. Rev. Lett. 107, 155501 (2011).

${ }^{17}$ T. D. Nguyen, E. Jankowski, and S. C. Glotzer, ACS Nano 5, 8892 (2011).

${ }^{18}$ P. F. Damasceno, M. Engel, and S. C. Glotzer, Science 337, 453 (2012).

${ }^{19}$ S. Torquato and Y. Jiao, Phys. Rev. E 86, 011102 (2012).

${ }^{20}$ M. R. Khadilkar and F. A. Escobedo, J. Chem. Phys. 137, 194907 (2012).

${ }^{21}$ R. Gabbrielli, Y. Jiao, and S. Torquato, Phys. Rev. E 86, 041141 (2012).

${ }^{22}$ U. Agarwal and F. A. Escobedo, J. Chem. Phys. 137, 024905 (2012).

${ }^{23}$ S. Atkinson, Y. Jiao, and S. Torquato, Phys. Rev. E 86, 031302 (2012).

${ }^{24}$ R. Blaak and B. M. Mulder, Phys. Rev. E 58, 5873 (1998).

${ }^{25}$ K. J. Strandburg, Rev. Mod. Phys. 60, 161 (1988).

${ }^{26}$ J. G. Dash, Rev. Mod. Phys. 71, 1737 (1999).

${ }^{27}$ S. Furukawa and S. De Feyter, Two-Dimensional Crystal Engineering at the Liquid-Solid Interface, Topics in Current Chemistry Vol. 287 (SpringerVerlag, Berlin/Heidelberg, 2009).

${ }^{28}$ Z. Quan and J. Fang, Nano Today 5, 390 (2010).

${ }^{29}$ D. Frenkel and R. Eppenga, Phys. Rev. A 31, 1776 (1985).

${ }^{30}$ M. C. Lagomarsino, M. Dogterom, and M. Dijkstra, J. Chem. Phys. 119, 3535 (2003).

${ }^{31}$ M. A. Bates and D. Frenkel, J. Chem. Phys. 112, 10034 (2000).

${ }^{32}$ J. A. Cuesta and D. Frenkel, Phys. Rev. A 42, 2126 (1990).

${ }^{33}$ T. Schilling, S. Pronk, B. Mulder, and D. Frenkel, Phys. Rev. E 71, 036138 (2005). 
${ }^{34}$ K. W. Wojciechowski and D. Frenkel, Comput. Methods Sci. Technol. 10, 235 (2004); access online at http://www.man.poznan.pl/cmst/www. old/papers/10_2/CMST10-12.pdf.

${ }^{35}$ K. Zhao and T. G. Mason, Phys. Rev. Lett. 103, 208302 (2009).

${ }^{36}$ A. Donev, J. Burton, F. H. Stillinger, and S. Torquato, Phys. Rev. B 73, 054109 (2006).

${ }^{37}$ K. Zhao, R. Bruinsma, and T. G. Mason, Proc. Natl. Acad. Sci. U.S.A. 108, 2684 (2011).

${ }^{38}$ C. Avendano and F. A. Escobedo, Soft Matter 8, 4675 (2012).

${ }^{39}$ J. M. Kosterlitz and D. J. Thouless, J. Phys.: Condens. Matter 6, 1181 (1973).

${ }^{40}$ D. R. Nelson and B. I. Halperin, Phys. Rev. B 19, 2457 (1979).

${ }^{41}$ A. P. Young, Phys. Rev. B 19, 1855 (1979).

${ }^{42}$ E. P. Bernard and W. Krauth, Phys. Rev. Lett. 107, 155704 (2011).

${ }^{43}$ S. C. Glotzer, Nature (London) 481, 450 (2012).

${ }^{44}$ L. Filion, M. Marechal, B. van Oorschot, D. Pelt, F. Smallenburg, and M. Dijkstra, Phys. Rev. Lett. 103, 188302 (2009).

${ }^{45}$ J. de Graaf, L. Filion, M. Marechal, R. van Roij, and M. Dijkstra, J. Chem. Phys. 137, 214101 (2012).

${ }^{46}$ C. J. DeSantis and S. E. Skrabalak, Langmuir 28, 9055 (2012).

${ }^{47}$ B. Widom, J. Chem. Phys. 39, 2808 (1963).
${ }^{48}$ D. Frenkel and B. Smit, Understanding Molecular Simulation, 2nd ed. (Academic Press, Inc., 2001).

${ }^{49}$ D. Frenkel and A. J. C. Ladd, J. Chem. Phys. 81, 3188 (1984).

${ }^{50}$ D. Frenkel and B. M. Mulder, Mol. Phys. 55, 1171 (1985).

${ }^{51}$ C. Vega and E. G. Noya, J. Chem. Phys. 127, 154113 (2007).

${ }^{52}$ U. Agarwal and F. A. Escobedo, Nature Mater. 10, 230 (2011).

${ }^{53}$ See supplementary material at http://dx.doi.org/10.1063/1.4799269 for movie files visualizing the melting that occurs at the boundary of the square crystal and isotropic liquid.

${ }^{54}$ A. Dong, X. Ye, J. Chen, and C. B. Murray, Nano Lett. 11, 1804 (2011).

${ }^{55}$ A. Dong, J. Chen, P. M. Vora, J. M. Kikkawa, and C. B. Murray, Nature (London) 466, 474 (2010).

${ }^{56}$ W. H. Evers, B. Goris, S. Bals, M. Casavola, J. de Graaf, R. van Roij, M. Dijkstra, and D. Vanmaekelbergh, "Low-dimensional semiconductor superlattices formed by geometric control over nanocrystal attachment," Nano Lett. (in press).

${ }^{57}$ J. M. Polson, E. Trizac, S. Pronk, and D. Frenkel, J. Chem. Phys. 112, 5339 (2000).

${ }^{58}$ H. N. W. Lekkerkerker and R. Tuinier, Depletion Interaction, Lecture Notes in Physics Vol. 833 (Springer, Berlin, 2011). 